\title{
The Real-time Effect of Public Health Interventions on the COVID-19 Epidemic in Hubei Province
}

\author{
Jiamin Liu ${ }^{1}, Z_{\text {e }}$ Chen $^{1}$, Jianqiang Zhang ${ }^{1}$, Yanyan Ouyang ${ }^{1}$, Xu Guo ${ }^{2}$, and Wangli \\ $\mathrm{XU}^{* 1,3}$ \\ ${ }^{1}$ School of Statistics, Renmin University of China, Beijing, China \\ ${ }^{2}$ School of Statistics, Beijing Normal University, Beijing, China \\ ${ }^{3}$ Center for Applied Statistics, Renmin University of China, Beijing, China
}

\begin{abstract}
The Corona Virus Disease 2019 (COVID-19) emerged in Wuhan, China in December 2019. In order to control the epidemic, the Chinese government adopted several public health measures. To study the influence of these measures on the transmissibility of COVID-19 in the city of Wuhan and other cities in the Hubei province, China, we establish generalized semi-varying coefficient models for the number of new diagnosed cases and estimate the varying coefficient for the covariates by the spline method. Since the pandemic was most severe in Wuhan, we fitted separate models for Wuhan and the remaining 16 cities in Hubei. Estimators for the incubation periods, the real-time transmission rates, and the real-time reproduction numbers were obtained. The results demonstrate that the changes in the real-time transmission rate in Wuhan and other cities in Hubei are almost simultaneous. Futher, public health interventions such as restriction of traffic, adjustment of the diagnosed standard, deployment of medical resources, and improvement of nucleic acid testing capacity, had positive effects on reducing the transmission of COVID-19.
\end{abstract}

Keywords generalized semi-varying coefficient model; incubation time; time-dependent reproduction number; transmission rate

\footnotetext{
${ }^{*}$ Corresponding author. Email: wlxu@ruc.edu.cn.
} 


\title{
公共卫生措施对湖北省新冠肺炎疫情的实时影响
}

\author{
刘佳敏 ${ }^{1}$ ，陈泽 ${ }^{1}$, 张建强 ${ }^{1}$, 欧阳妍妍 ${ }^{1}$, 郭旭 ${ }^{2}$, 许王莉*1,3 \\ 1 中国人民大学统计学院 \\ 2 北京师范大学统计学院 \\ 3 中国人民大学应用统计科学研究中心
}

\begin{abstract}
摘要
2019 年 12 月, 湖北省出现了新型冠状病毒肺炎（COVID-19）疫情。为了控制疫情传播，中国政 府积极采取了一系列公共卫生干预措施。本文采用广义半变系数模型对新增确诊人数建立模型, 采用样条方法估计各协变量的系数以评估公共卫生干预措施对湖北省新冠肺炎疫情防控的实时 影响。考虑到武汉市疫情的严重性，本文针对武汉市和湖北省其他市分别构建模型。并基于模型 估计结果，分别估计了武汉市和湖北其他地区新冠肺炎的潜伏期、实时传播率以及实时再生数。 研究结果表明 COVID-19 病毒传播率在武汉市及湖北省其他地区的变化基本是同步的，并且交 通限制、调整确诊标准、调配医疗资源以及提升核酸检测能力等措施对于湖北省 COVID-19 疫 情的控制发挥了积极作用。
\end{abstract}

关键词 广义半变系数模型; 潜伏期; 实时传播率; 实时再生数

\section{1 引言}

2019 年 12 月 31 日，武汉市卫健委首次通报了 27 例不明原因的病毒性肺炎病例。由于春 节期间的人口流动，疫情迅速蔓延到全国各地。2020 年 2 月 11 日，世界卫生组织（WHO）正 式将该疾病命名为 2019 冠状病毒病 (Corona Virus Disease 2019, 简称 COVID-19)，与此同时， COVID-19 在亚洲（日本、韩国），欧洲（英国、西班牙、意大利等）和美国快速传播。WHO 于 2020 年 3 月 11 日正式将新冠病毒定性为全球大流行。截至 2020 年 5 月 31 日，国家卫健委累 计报告确诊病例 83107 例，其中死亡病例 4634 例。湖北省确诊病例 68135 例，死亡病例 4512 例。武汉市确诊病例 50340 例，死亡病例 3869 例。

新冠疫情爆发以来，武汉市采取了交通限制、“封城”、社会隔离、佩戴口罩、社区检疫和限 制聚会等措施。并且自 2020 年 2 月 13 日起, 将临床诊断病例数纳入确诊病例数公布。Lin et al. (2020) 将这些措施归纳为公共卫生干预措施 (Public Health Interventions)，其研究结果表明在 个人和社会层面实施的公共卫生干预措施可有效控制 COVID-19 在武汉及其他省份的传播。随 后其他城市陆续启动重大公共卫生事件一级响应，实施严格的公共卫生干预措施来控制疫情传 播, 这些非常规公共卫生干预措施对于疫情的控制起了决定性的作用 (Kraemer et al., 2020)。《中 国一世界卫生组织新型冠状病毒肺炎（COVID-19）联合考察报告》指出，武汉市发生新冠疫 情后，党中央以 “控制感染源，阻断传播、积极救治、减少死亡”为主要策略，采取了研发检测 试剂盒、统筹调配物资、新建医院、启用储备床位和征用相应场所等措施。据《长江日报》报道,

*通讯作者。电子邮箱：wlxu@ruc.edu.cn。 
在 2020 年 1 月 16 日之前, 湖北省的核酸检测样本需要由北京市指定的检测机构检测。采样之 后，通常需要 3-5 天才能收到反馈结果，武汉市每天仅能检测 200 多个样本。到 2020 年 1 月 22 日左右，每天接受核酸检测的人数大约为 2000 人，收到反馈结果的时间也大约缩短至 2 天。据 央视新闻报道，到 2020 年 2 月 5 日，核酸检测时间缩短为 1 天，武汉市每天的检测样本数量 达到 6500 个。国家卫健委在新闻发布会上也提到，自 2020 年 2 月 8 日起，从采样送检到收到 反馈结果仅需 4-6 小时。截至 2 月 28 日，核酸检测能力从每天 200 人份提高到 2 万人份。可 见，核酸检测能力有了很大提升。自 2020 年 1 月 24 日起，国家卫建委从全国范围内调配医疗 队伍及医疗物资支援武汉等湖北重点地区的医疗救治工作。截至 2020 年 3 月 17 日，共有来自 全国各地的 300 多支医疗队，约 4.5 万名医护人员驰援湖北。为收治不断增多的新冠肺炎患者， 疫情重灾区武汉市将 40 多家医院设立为新冠肺炎定点医院, 建立的 13 所方舱医院提供了万余 张床位。据湖北省卫健委报道, 2020 年 2 月 4 日 0-24 时, 湖北省新增新型冠状病毒感染的肺炎 病例 3156 例，新增死亡 65 例，新增出院 125 例。 2020 年 2 月 25 日 0-24 时，湖北省新增新型 冠状病毒感染的肺炎病例 401 例, 新增死亡 52 例, 新增出院 2058 例。可见公共卫生干预措施 实施以来, 湖北省的新增确诊人数和死亡人数明显下降, 新增治愈人数在持续增加, 这些数据也 说明公共卫生干预措施有效遏制了湖北省新冠疫情的进一步蔓延。基于此，本文从交通限制、调 整确诊标准、调配医疗资源以及提升核酸检测能力四个方面研究公共卫生干预措施对湖北省疫 情防控的影响。

“封城” 是实现 “交通限制” 的主要措施之一，且交通限制的实施有助于减少人口流动，从 而控制感染源、减少病毒输出。因此许多文献对 COVID-19 传播和人口流动、交通限制以及 “封 城”之间的关系进行了研究。Tian et al. (2020) 指出, 武汉市的 “封城” 措施使 COVID-19 疫 情在其他城市开始爆发的时间推迟了 2.91 天。与较晚开始控制的城市相比，提早实施控制措施 的城市在其疫情爆发后的第一周报告的病例数更少。Kraemer et al. (2020) 阐释了人口流动对全 国范围内 COVID-19 病例传播和空间分布的影响。他们的研究结果表明严格的控制措施有效减 缓了 COVID-19 的传播。同时他们还估计了潜伏期和疫情倍增时间。Zhao et al. (2020) 研究了 每天从武汉到其他省份的乘客数量与确诊病例数之间的关系, 发现从武汉市出发的乘客人数乘 以武汉市的感染率与目的地报告的病例数之间存在正相关关系。此外，还有一些文献对其传播 模式和流行病学参数（包括基本再生数, 潜伏期, 倍增时间等）进行了研究。例如, Wu et al. (2020) 基于 2019 年 12 月 31 日至 2020 年 1 月 28 日从武汉市输出的病例数, 采用敏感-暴露-感 染-康复（SEIR）模型估算了 2019 年 12 月 1 日至 2020 年 1 月 25 日武汉市的感染人数。同 时他们还估计了基本再生数和武汉市向国内其他城市输入的病例数。通过使用 2020 年 1 月 20 日至 28 日在武汉市以外的地区出现的 88 例确诊病例的旅行史、症状发作时间等数据, Backer et al. (2020) 采用威布尔分布、伽玛分布和对数正态分布三种参数形式来拟合潜伏期。Tuite et al. (2020) 构建了一个疾病传播模型来估计 2019 年 11 月中旬以来的累计感染病例数。模型估计的 确诊病例数与报告病例数的差异表明将基本再生数减少到 1.5 是合理的。Du et al. (2020) 估计 出 COVID-19 病毒的潜伏期为 5-6 天，发病到确诊时长为 4-5 天。他们还估计了 2020 年 1 月 23 日之前 COVID-19 病例从武汉向国内其他城市的转移概率以及疫情倍增时间。

上述已有的文献重点研究了 “封城” 或 “交通限制” 这一措施对疫情控制的影响，而对于调 整确诊标准、调配医疗资源、提升核酸检测能力等公共卫生干预措施对控制疫情传播的影响有待 
量化。且这些措施对疫情控制的影响在疫情发展的不同阶段是变化的, 因此本文拟采用广义半变 系数模型对新增确沴人数分别建立模型, 采用样条方法估计各协变量随时间变化的系数以评估 公共卫生干预措施对湖北省新冠肺炎疫情防控的实时影响。此外，对潜伏期、实时传播率和实时 再生数等关键指标的进一步研究, 有助于防疫部门了解疫情的传播模式, 这对新冠肺炎防控决策 具有重要的参考价值。因此, 基于广义半变系数模型估计结果, 本文分别估计新冠病毒在武汉市 和湖北其他城市的潜伏期、实时传播率以及实时再生数等关键指标, 以期为防疫部门制定相关政 策措施提供理论参考。

\section{2 疫情数据模型拟合与分析}

为评估封城、核酸检测技术改进和医护人员增援等公共卫生干预措施对各市疫情防控的影 响, 本文基于不同的协变量, 分别对武汉和其他 16 个城市每天的新增确诊病例数建立模型, 并 估计新冠肺炎在武汉市和其他 16 个城市的潜伏期、实时传播率和实时再生数。据报道，在 2020 年 1 月 16 日之前，湖北省没有核酸检测试剂盒。因此武汉市的所有分析基于 2020 年 1 月 16 日至 3 月 10 日的数据进行。同时, 神农架林区于 2020 年 1 月 28 日首次出现确诊病例, 所以湖 北其他地区的所有分析基于 2020 年 1 月 28 日至 3 月 10 日的数据进行。与湖北其他城市相比, 武汉市人口规模大，且是我国重要的交通枢纽，大批人员流动加快了病毒的传播。且 2020 年 2 月 29 日发布的《中国一一世界卫生组织新型冠状病毒肺炎（COVID-19）联合考察报告》指出， 在紧邻武汉的地区传播程度低于武汉, 而湖北省其他地区的动力学与全国其他地区更为接近。但 对于其他地区尚未给出明确划分，因此本文将其他 16 个城市作为整体进行分析。

\section{1 变量定义及符号说明}

设下标 $t$ 表示第 $t$ 天，下标 $k$ 表示湖北省的第 $k$ 个城市，其中 $k=1$ 表示武汉。表 1 介绍了 本文用到的符号及其含义。表 1 中, 下标为 $t$ 的变量用于武汉的分析，下标为 $k t$ 的变量在 $k \neq 1$ 时用于除武汉市外湖北省其他城市的分析。 $B C_{t}$ 为类别变量，指武汉市是否封城。变量 $E I_{k t}$ 表 示从武汉市到湖北省 $k$ 市的迁徙强度，因此变量 $E I_{k t}$ 在一定程度上也反映了武汉市是否封城。 $C D_{t}$ 为类别变量，指确诊人数是否包含临床诊断病例数。据湖北省卫健委报道，自 2020 年 2 月 13 日起，临床诊断病例数也纳入确诊病例数公布。武汉市的确诊病例数从 2 月 12 日的 19558 例攀升至 2 月 13 日的 32994 例。确诊标准的调整可能是导致确诊数激增的原因之一，因此，在 武汉模型中考虑了分类变量 $C D_{t}$ 。但由于湖北省其他地区的确诊人数不像武汉市迅速增长且疫 情不及武汉市严峻, 因此调整确诊标准对其他城市确诊人数的影响可能较小。因此, 在湖北省其 他 16 个城市的模型中未考虑分类变量 $C D_{t 。}$ 考虑到湖北省其他 16 个城市核酸检测所需的平均 时间与武汉市大致相同，因此在其他 16 个城市的模型中，核酸检测时间 $T N_{t}$ 也同样适用。协 变量 $N C_{t}$ 和 $N N_{t}$ 表示武汉市定点医院病床数和每天核酸检测数，由于湖北省其他城市尚未公 布相关信息，因此对于湖北省其他城市这部分数据尚未收集到。同时考虑到对于湖北其他城市而 言，床位数和核酸试剂盒并不是极度紧缺，因此针对湖北省其他 16 个城市构建的模型中，本文 没有考虑定点医院床位数和核酸检测数量两个变量。 
表 1: 模型的符号定义。

\begin{tabular}{|c|c|}
\hline 符号 & 含义 \\
\hline$Y_{k t}$ & 截至第 $t$ 天，城市 $k$ 的累计确诊人数 \\
\hline$B C_{t}$ & 在第 $t$ 天，武汉市是否封城， 2020 年 1 月 23 日之前该变量为 0 ，其余为 1 \\
\hline$E I_{k t}$ & 在第 $t$ 天，从武汉市到城市 $k$ 的迁徙强度 \\
\hline$C D_{t}$ & $\begin{array}{l}\text { 在第 } t \text { 天, 确诊人数是否包含临床诊断病例数, } \\
2020 \text { 年 } 2 \text { 月 } 13 \text { 日之前该变量为 } 0 \text {, 其余为 } 1\end{array}$ \\
\hline$N C_{t}$ & 截至第 $t$ 天，武汉市定点医院的累计床位数 \\
\hline$N D_{k t}$ & 截至第 $t$ 天，支援城市 $k$ 的累计医护人员数 \\
\hline$N N_{t}$ & 武汉市第 $t$ 的核酸检测样本数 \\
\hline$T N_{t}$ & 在第 $t$ 天，核酸检测从采样送检到反馈结果所需的平均时间 \\
\hline$W_{k t}$ & 截至第 $t$ 天，城市 $k$ 的累计感染人数 \\
\hline$m_{1}$ & COVID-19 病毒在武汉市的潜伏期 \\
\hline$m_{2}$ & COVID-19 病毒在除武汉市外湖北省其他地区的潜伏期 \\
\hline$\gamma_{1 t}$ & 在第 $t$ 天，COVID-19 在武汉市的传播率，定义为 $d W_{k t} /\left(W_{k, t-1}-W_{k, t-m-1}\right)$ \\
\hline$\gamma_{t}$ & 在第 $t$ 天，COVID-19 在除武汉市外湖北省其他地区的传播率, \\
\hline & 定义为 $\sum_{k=2}^{K}\left(d W_{k t}\right) / \sum_{k=2}^{K}\left(W_{k, t-1}-W_{k, t-m-1}\right)$ \\
\hline$R_{1 t}$ & 武汉市第 $t$ 天的再生数 \\
\hline $\bar{R}_{t}$ & 除武汉市外湖北省其他地区第 $t$ 天的再生数 \\
\hline
\end{tabular}

\section{2 广义半变系数模型对新增确诊人数建模}

为评价这些公共卫生干预措施对疫情防控的实时影响, 本文拟采用广义半变系数模型对武汉 市及湖北省其他市的新增确诊人数进行建模分析。广义变系数模型的一般形式为 (Hastie et al., 1993):

$$
Y=g\left\{X^{\mathrm{T}} \beta(U)\right\}+\varepsilon,
$$

其中, $g(\cdot)$ 为已知的链接函数, $(X, U)$ 为协变量, $\beta(U)=\left\{\beta_{1}(U), \ldots, \beta_{p}(U)\right\}^{\mathrm{T}}$ 是未知的回归系 数, $\varepsilon$ 为误差项且满足 $E(\varepsilon \mid X, U)=0$ 。可见回归系数 $\beta(U)$ 能够更好地反映协变量 $X$ 对响应 变量 $Y$ 的影响如何随协变量 $U$ 变化, 对于协变量 $(X, U)$ 和响应变量 $Y$ 间的关系更具有解释 性。因此, 广义变系数模型已被广泛用于分析纵向数据 (Hoover et al., 1998)、函数型数据 (Rice et al., 1991) 及时间序列数据 (Huang et al., 2004)。对于系数 $\beta(U)$ 的估计，文献中一般采用核 或局部多项式 (Wu et al., 1998)、光滑样条 (Chiang et al., 2001) 或 B 样条 (赵培信, 2013) 等估 计方法。

本文假设第 $k$ 个城市在第 $t$ 天的新增病例数 $\left(d Y_{k t}\right)$ 服从泊松分布，即

$$
d Y_{k t}=Y_{k t}-Y_{k, t-1} \sim \operatorname{Poisson}\left(\lambda_{k t}\right) 。
$$

论文假设武汉市新增确沴人数的模型为:

$$
\log \left(\lambda_{1 t}\right)=\beta_{0}+\beta_{B C} B C_{t}+\beta_{C D} C D_{t}+\beta_{N N}(t) N N_{t}+\beta_{T N}(t) T N_{t}+\beta_{N D}(t) N D_{1 t \circ}
$$


模型(1)是一个广义半变系数模型。考虑到核酸检测技术和医护人员总数对疫情控制的影响是随 时间变化的, 而武汉是否封城 $\left(B C_{t}\right)$ 和是否增加临床诊断 $\left(C D_{t}\right)$ 两个分类变量对疫情的影响与 时间无关。因此，在模型 $(1)$ 中将 $\beta_{N N}(t), \beta_{T N}(t)$ 以及 $\beta_{N D}(t)$ 设为依赖于时间的系数，而 $\beta_{B C}$ 和 $\beta_{C D}$ 为常系数。

在湖北省其他 16 个城市新增确诊人数的模型中，本文仅考虑迁徙强度 $\left(E I_{k t}\right)$ 和医疗人员 总数 $\left(N D_{k t}\right)$ 对于新增确诊病例数的影响。与武汉市相比, 其他城市确诊病例数量增长较为缓 慢, 所以在模型中不考虑与核酸检测速度和确诊标准调整相关的变量。同时, 论文假设每个协变 量对 16 个城市的疫情控制具有相同的影响，并且其影响程度随时间变化。因此，本文假设湖北 省其他 16 个城市新增确诊人数的模型为:

$$
\log \left(\lambda_{k t}\right)=\beta_{0}+\beta_{E I}(t) E I_{k t}+\beta_{N D}(t) N D_{k t}, \text { 对于第 } k \text { 个城市, } k=2, \ldots, K_{\circ}
$$

\section{3 数据来源}

本文数据主要来自湖北省卫健委网站发布的相关数据和新闻，以及百度迁徙网站发布的人 口迁移数据。首先，从湖北省卫健委网站上收集到 2020 年 1 月 11 日至 3 月 17 日湖北省各市 的新冠肺炎确诊和死亡病例数，包括武汉、黄石、十堰、宜昌、襄阳、鄂州、荆门、孝感、荆州、 黄冈、咸宁、随州、恩施、仙桃、潜江, 天门和神农架 17 个城市或地区。为了考察武汉市输出病 例对其他地区疫情传播的影响，本文从百度迁徙（http://qianxi.baidu.com）网站上收集了 2020 年 1 月 16 日至 31 日从武汉市到其他 16 个城市的人口迁移数据，包括武汉市的迁徙规模指数 和迁入湖北其他各城市的比例。参考Chen et al. (2020) 的方法, 本文用武汉市的迁徙规模指数 乘以湖北某市的比例来衡量从武汉市到该市的迁徙强度。

从中国各省市卫健委网站上，检索到援鄂医疗队的数量和到达时间。数据显示，2020 年 1 月 24 日至 3 月 17 日，全国共计派出 250 支医疗队、约 3 万名医护工作者援助武汉市，其他 63 支医疗队分别对口支援湖北省的其他 16 个城市。图 1 展示了截至 3 月 17 日，除武汉市外湖北 省其他 16 个城市的医护人员分布情况。

本文还从武汉市卫健委网站上收集到 2020 年 1 月 31 日至 2 月 25 日武汉市定点医院每天 的床位数。该网站显示，截至 2020 年 1 月 31 日，武汉市已有 23 家医院被指定为收治发热病 人的定点医院。首批定点医院是 2020 年 1 月 16 日之前就已投入使用的武汉市金银潭医院和武 汉肺科医院，约提供床位 800 张。第二批定点医院包括汉口医院，武昌医院，武汉市第五医院 等 10 家医院，提供约 3800 张床位，并于 2020 年 1 月 22 日开始收治 COVID-19 患者。第三批 定点医院是同济医院中法新城院区，武汉协和医院西院，武汉市第六医院等 14 家医院。从 2020 年 1 月 27 日至 31 日，第三批定点医院中共有 11 家医院收治 COVID-19 患者，约提供 2000 张 床位。根据上述信息，本文对 2020 年 1 月 16 日至 31 日武汉市定点医院的病床数进行了补充， 完整数据如图2 (a) 所示。

本文从武汉市卫健委网站上收集到 2020 年 2 月 19 日至 3 月 17 日武汉市每天的核酸检测 人数。根据这些新闻信息，本文通过插值得到 2020 年 2 月 19 日前，武汉市每天的检测样本数。 图2 (b) 展示了 2020 年 1 月 16 日至 3 月 17 日武汉市每天的核酸检测数。同时，截至 2020 年 3 月 17 日，核酸检测时间在四个时间段有三次缩减，分别是：3-5 天（2020 年 1 月 16 日之前), 


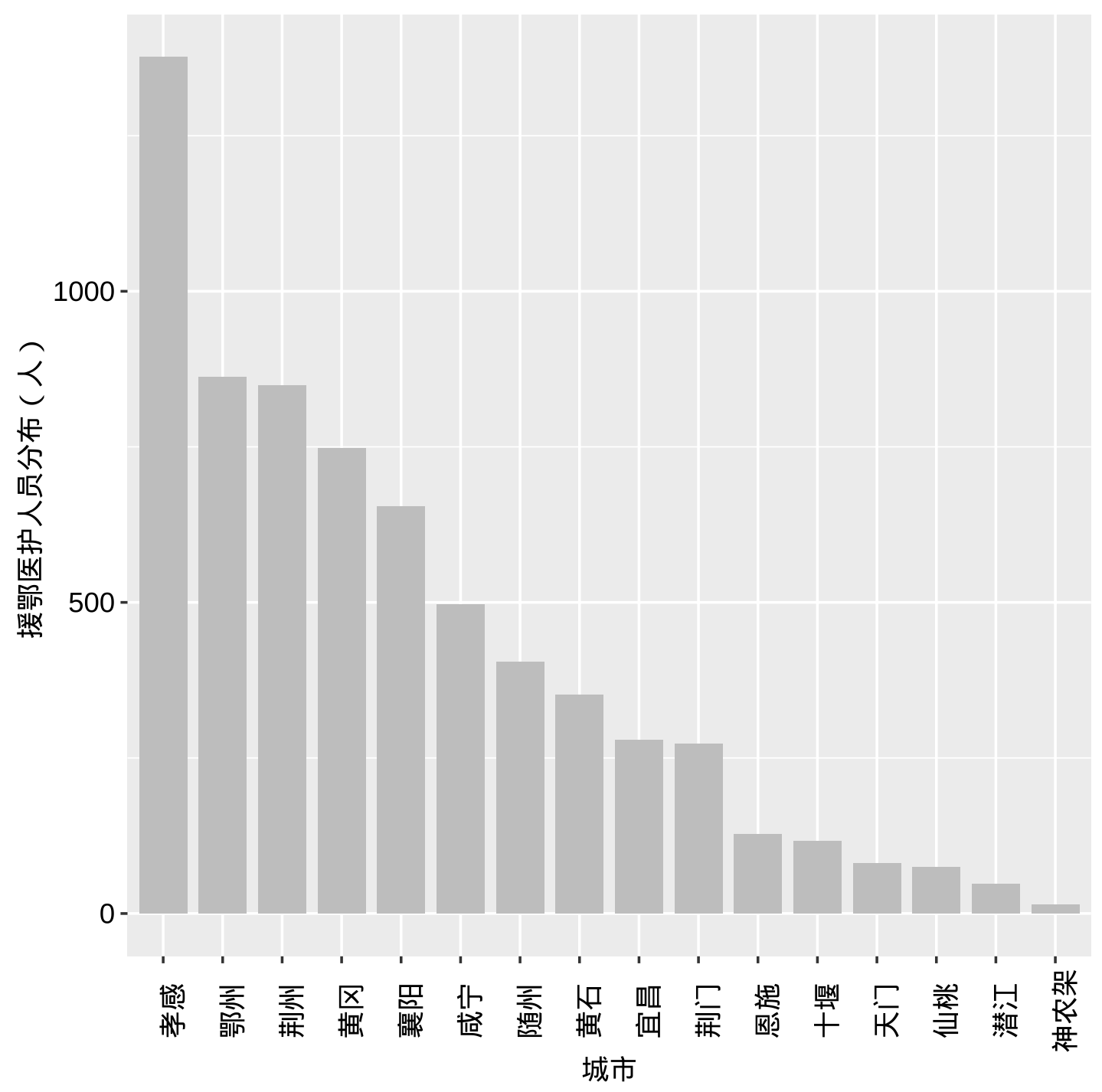

图 1: 除武汉市外湖北省其他 16 个城市的医护人员分布（截至 2020 年 3 月 17 日）。

2 天（自 2020 年 1 月 16 日起)， 1 天（自 2020 年 2 月 4 日起)，4-6 小时（自 2020 年 2 月 8 日起)。

\subsection{COVID-19 潜伏期和实时再生数的计算}

本文将截至 2020 年 1 月 16 日，武汉市感染 COVID-19 病毒的累计人数记为 $N_{1,0}$; 截至 2020 年 1 月 28 日，除武汉市外湖北省其他城市感染 COVID-19 病毒的累计人数分别记为 $N_{k, 0}$, $k=2, \cdots, K$, 且 $N_{1,0}$ 和 $N_{k, 0}$ 为常数。并假设 COVID-19 病毒的潜伏期为 $m$ 天。根据已有的 研究结果, 我们将潜伏期 $m$ 的取值范围设为 $[1,30]$ 天。因此, 第 $k$ 个城市在第 $t-m$ 天的平均 新增感染病例将在第 $t$ 天转换为平均新增确诊病例, 即

$$
E\left(d Y_{k t}\right)=E\left(d W_{k, t-m}\right)_{\circ}
$$


(a)

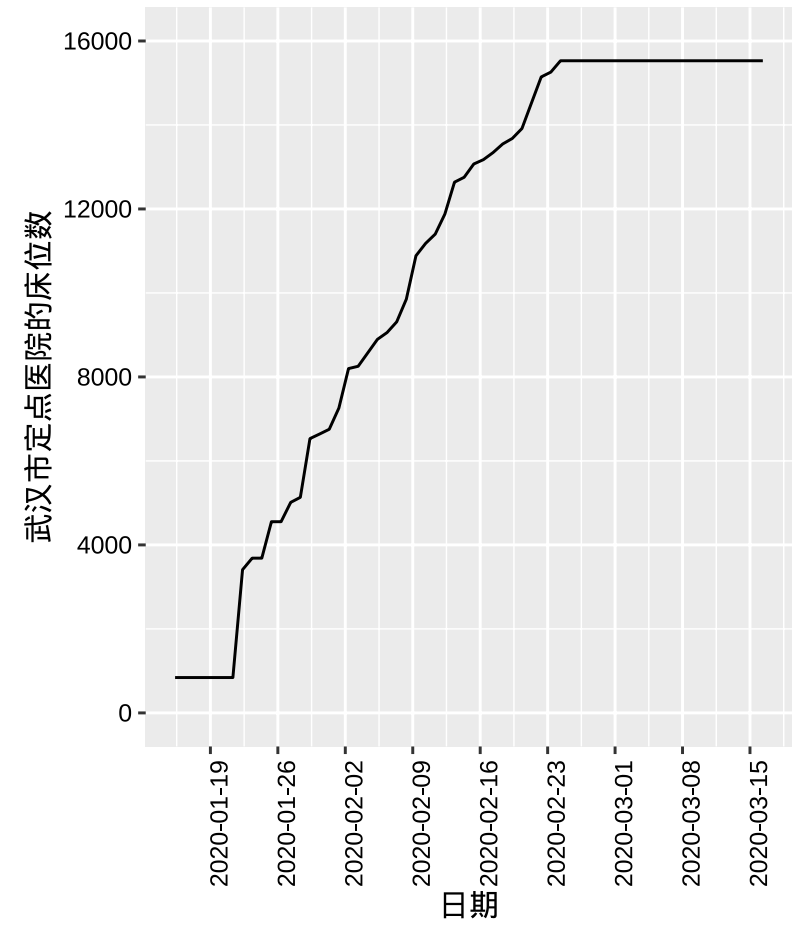

图 2: (a) 武汉市每日核酸检测人数。 (b)

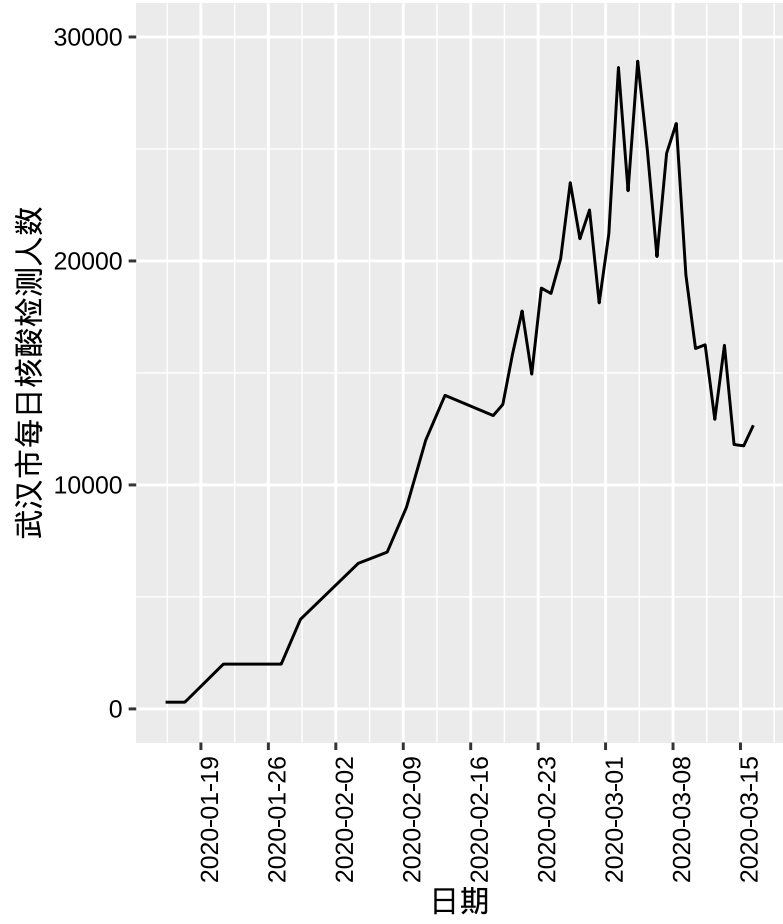

(b) 武汉市定点医院的床位数。

根据式(3)，有

$$
E\left(W_{k, t-m}\right)-E\left(W_{k, t-m-1}\right)=\lambda_{k t \circ}
$$

对于城市 $k=1, \cdots, K$ 和时间 $t=1, \cdots, T$ ，本文将模型(1) 和(2)中 $\lambda_{k t}$ 的估计值记为 $\widehat{\lambda}_{k t \circ}$ 同 时， $N_{k, 0}$ 的定义表明 $W_{k, 0}=N_{k, 0}$ 。 因此，对于给定的 $m_{1}, m_{2}$ 和 $N_{k, 0}$, 本文通过简单计算分 别估计出截至 $t-m_{1}$ 天武汉市和 $t-m_{2}$ 天湖北其他城市感染 COVID-19 病毒的累计人数, 并 把相应的估计值分别记为 $\widehat{W}_{1, t-m_{1}}$ 和 $\widehat{W}_{k, t-m_{2}}$, 即

$$
\begin{aligned}
& \widehat{W}_{1, t-m_{1}}=N_{1,0}+\sum_{j=m_{1}+1}^{t} \widehat{\lambda}_{1 j}, \text { 对于武汉市, } \\
& \widehat{W}_{k, t-m_{2}}=N_{k, 0}+\sum_{j=m_{2}+1}^{t} \widehat{\lambda}_{k j}, \text { 对于第 } k \text { 个城市 } k=2, \cdots, K_{\circ}
\end{aligned}
$$

根据式(3)可知，对于武汉市而言，基于合理的潜伏期 $m_{1}$ 估计得到的 $\widehat{Y}_{1 t}$ 和 $\widehat{W}_{1, t-m_{1}}$ 应该较为 接近，且对于湖北其他市也类似。因此，论文按照以下准则，从集合 $\{1,2, \cdots, 30\}$ 中得到潜伏 
期的最优估计值 $\widehat{m}_{1}$ 和 $\widehat{m}_{2}$,

$$
\begin{aligned}
& \widehat{m}_{1}=\underset{m_{1}}{\arg \min }\left(T-m_{1}-1\right)^{-1} \sum_{t=m_{1}+1}^{T}\left(\widehat{Y}_{1 t}-\widehat{W}_{1, t-m_{1}}\right)^{2}, \\
& \widehat{m}_{2}=\underset{m_{2}}{\arg \min }\left(T-m_{2}-1\right)^{-1} \sum_{t=m_{2}+1}^{T}\left\{\sum_{k=2}^{K}\left(\widehat{Y}_{k t}-\widehat{W}_{k, t-m_{2}}\right)\right\}^{2} \circ
\end{aligned}
$$

基于潜伏期的估计 $\widehat{m}_{1}$ 和 $\widehat{m}_{2}$ 以及传播率的定义，本文用 $\widehat{W}_{1, t-m_{1}}$ 和 $\widehat{W}_{k, t-m_{2}}$ 分别对 $\gamma_{1 t}$ 和 $\gamma_{t}$ 进行估计，并将相应的估计值分别记为 $\widehat{\gamma}_{1 t}$ 和 $\widehat{\gamma}_{t}$, 即

$$
\widehat{\gamma}_{1 t}=\frac{\widehat{W}_{1 t}-\widehat{W}_{1, t-1}}{\widehat{W}_{1, t-1}-\widehat{W}_{1, t-m_{1}-1}}, \quad \widehat{\gamma}_{t}=\frac{\sum_{k=2}^{K}\left(\widehat{W}_{k t}-\widehat{W}_{k, t-1)}\right.}{\sum_{k=2}^{K}\left(\widehat{W}_{k, t-1}-\widehat{W}_{k, t-m_{2}-1}\right)} 。
$$

由于 COVID-19 病毒在武汉市和湖北省其他城市的潜伏期分别为 $m_{1}$ 和 $m_{2}$, 因此, 1 例 COVID19 病毒感染者将导致武汉市第 $t$ 天的新增感染数为 $R_{1 t}=m_{1} \gamma_{1 t}$ 例 (Lin et al., 2020)。而在除 武汉市外的湖北省其他地区， 1 例 COVID-19 病毒感染者将导致第 $t$ 天新增 $\bar{R}_{t}=m_{2} \gamma_{t}$ 例感染 者。 $R_{1 t}$ 和 $\bar{R}_{t}$ 被称为实时再生数，相应的估计值可以通过 $\widehat{m}_{1} ， \widehat{m}_{2}, \widehat{\gamma}_{1 t}$ 和 $\widehat{\gamma}_{t}$ 获得。

\section{3 模型估计结果和相关指标的解释}

本文采用样条方法 (Schumaker, 2007) 对模型(1)和 (2)的变系数进行估计, 并通过 1000 次 自助法重抽样得到变系数的置信区间 (Efron, 1985, 2003)。图3-5分别展示了模型(1)和(2)的估计 结果, 由于系数值在某些时刻太大, 导致置信区间整体看起来很窄。因此, 为了更清晰地展示各 个系数随时间的变化，论文还绘制了相应时段置信区间的放大图。

\section{1 公共卫生措施对控制 COVID-19 疫情传播的积极影响}

对于武汉市，本文得出潜伏期的最优估计值为 $\widehat{m}_{1}=8$, 图3给出了 $\lambda_{1 t}$ 和 $Y_{1 t}$ 的估计。模 型(1)中变量 $B C_{t}$ 系数的估计值是 -20.50 , 其 $95 \%$ 置信区间为 $(-22.52,-18.29)$ 。这表明，自 2020 年 1 月 23 日起，武汉市交通管制或封闭措施有效控制了 COVID-19 在武汉市的传播。另 一个分类变量 $C D_{t}$ 的系数估计值是 2.17 , 其 $95 \%$ 置信区间为 $(2.10,2.25)$ 。这说明确诊标准 的调整使更多的感染者被及时发现并隔离，并且增加临床诊断病例后，新增确诊病例约增加了 7 倍。可见这两项措施从根本上抑制了疫情的传播，对控制新冠肺炎疫情在武汉市进一步蔓延起到 了至关重要的作用。

模型(1)中变系数 $\beta_{N N}(t), \beta_{T N}(t)$ 以及 $\beta_{N D}(t)$ 的置信区间和相应时段的放大图可见图 4 。 图4 (a) 显示武汉地区每天核酸检测人数的系数 $\beta_{N N}(t)$ 在 $t=20$ 时降到零。在新冠肺炎疫情爆 发初期，核酸检测技术的不断改进有助于发现潜在的感染病例，每天新增确诊病例数也随之增 加。然而, 当 $t>20$ 的时候, 系数 $\beta_{N N}(t)$ 是负值。这表明, 自 2020 年 2 月 5 日起, 基于检测 能力的提高, 政府部门更有效地隔离了感染者。因此, 虽然第 $t$ 天的核酸检测病例数在增加, 但 是第 $t$ 天新增病例数可能会减少。同理，核酸检测时间的系数 $\beta_{T N}(t)$ 具有相反的变化趋势。 
(a)

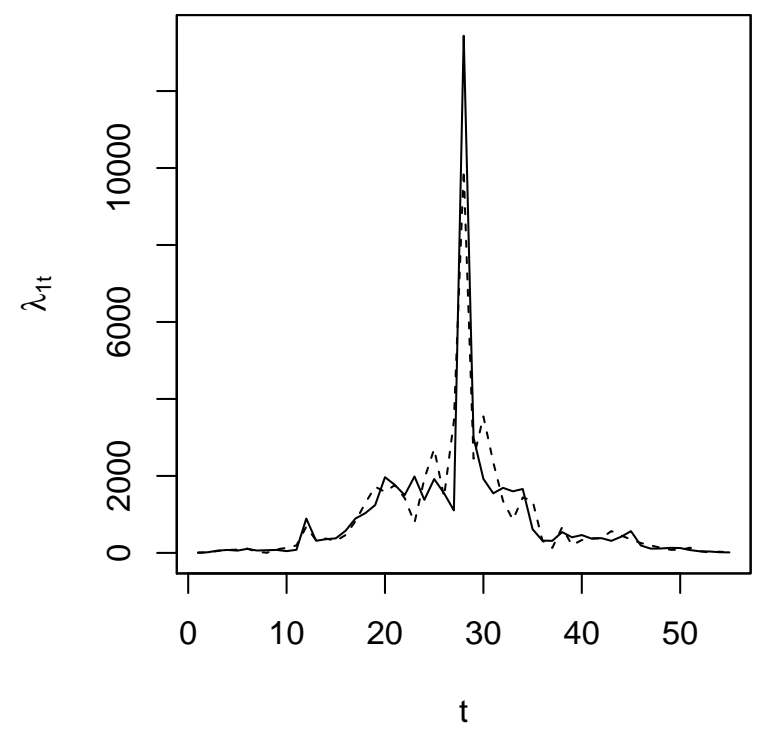

(b)

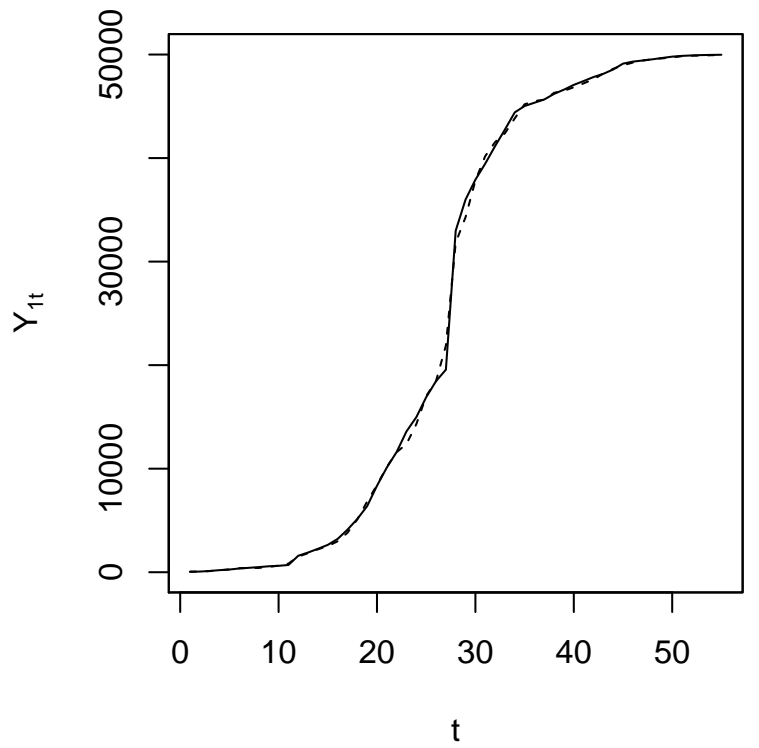

图 3: 武汉市新增确诊人数 $\left(\lambda_{1 t}\right)$ 和累计确诊人数 $\left(Y_{1 t}\right)$ 的估计。 (a) 分别用实线和虚线表示观测 到的 $\lambda_{1 t}$ 和估计的 $\lambda_{1 t}$; (b) 分别用实线和虚线表示观测到的 $Y_{1 t}$ 和估计的 $Y_{1 t}$ 。

在参与武汉救援的医护人员中，其中一部分被分配在多个核酸检测中心，另一部分被分配到 指定的治疗医院和隔离点。前者有助于提高武汉市的核酸检测能力, 而后者有助于降低武汉市民 的感染风险。图4 (a) 表明每日核算检测数 $N N_{t}$ 对 $d Y_{1 t}$ 的影响在疫情初期更为显著。因此, 类 似于系数 $\beta_{N N}(t)$, 医护人员数的系数 $\beta_{N D}(t)$ 是正数并且逐渐下降。2020 年 2 月 13 日, 新冠 肺炎确诊标准的调整使得超过 1.2 万例新冠肺炎病例被发现。而医护人员的不断增加, 使这些患 者逐渐被隔离或收治入院，同时其他市民的感染风险也大大降低。因此，从 $t=36 （ 2020$ 年 2 月 20 日）起，医护人员数的系数 $\beta_{N D}(t)$ 从 0 开始递减。

对于湖北其他城市，本文得出潜伏期的最优估计值为 $\widehat{m}_{2}=6$ 。为了展示模型 (2)的估计结 果, 针对十堰市, 图5 (a) 展示了所观测到的 $\lambda_{3 t}$ 和估计的 $\lambda_{3 t}$ 的图像, 图 $5(\mathrm{~d})$ 展示了观测到的 $Y_{3 t}$ 和估计的 $Y_{3 t}$ 的图像。图5 (b)-(c) 和 (e)-(f) 分别给出了变系数 $\beta_{E I}(t)$ 和 $\beta_{N D}(t)$ 的 $95 \%$ 置 信区间和相应时段的放大图。可以看出，在实施交通管制前，来自武汉的人口流动导致了湖北省 其他城市新增确诊病例的增加，以至于当 $t<20$ 时 $\beta_{E I}(t)$ 迁徙强度系数为正数。2020 年 1 月 23 日以后，武汉向湖北省其他城市的人口流动主要是医护人员和物资运输。因此，在 $t>20$ 时 (2020 年 2 月 5 日以后), 迁徙强度的系数为负数。同时，医护人员数的系数趋势与 $\beta_{E I}(t)$ 类似, 且迁徙强度系数 $\beta_{E I}(t)$ 和医护人员系数 $\beta_{N D}(t)$ 几乎同时从 0 开始下降。

\section{2 传播率和再生数的实时变化}

由于传播率和再生数的实时变化趋势一致，因此本文仅在图6 (a) 和 (b) 中分别展示武汉市 （2020 年 1 月 24 日至 3 月 2 日）实时再生数 $R_{1 t}$ 和湖北其他市（2020 年 2 月 3 日至 3 月 10 日）实时再生数估计值的变化情况以及 $95 \%$ 置信区间。可以看到，武汉市的再生数于 2020 年 1 
(a)

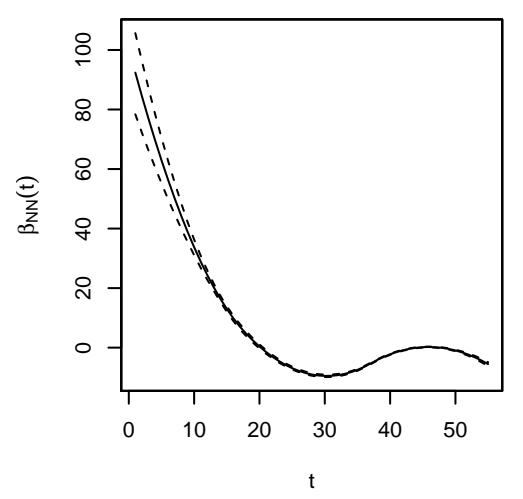

(d)

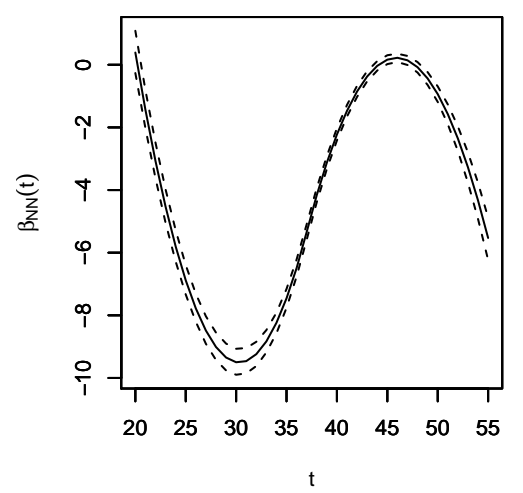

(b)

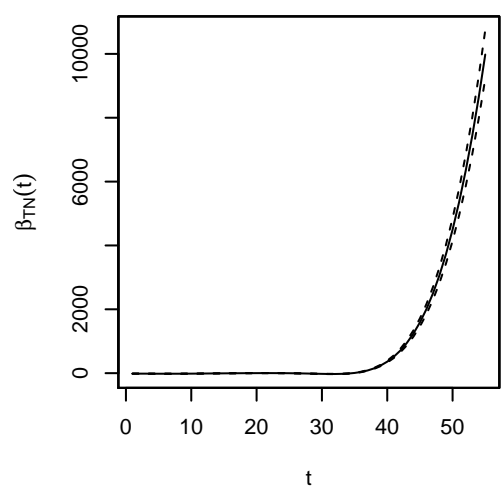

(e)

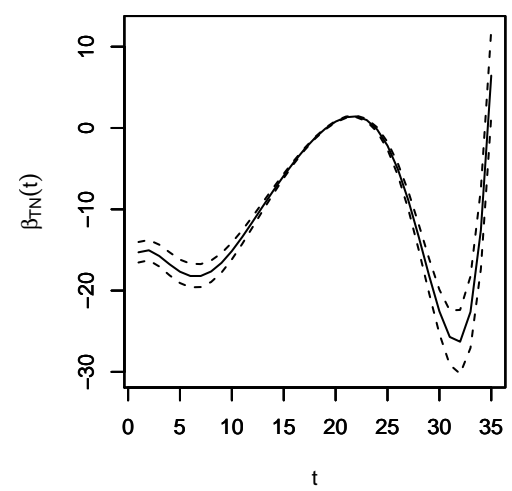

(c)

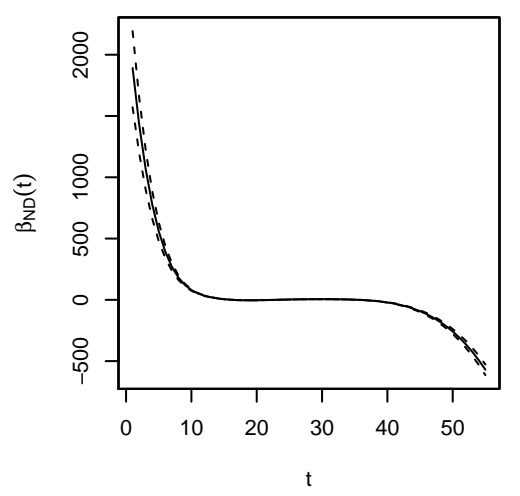

(f)

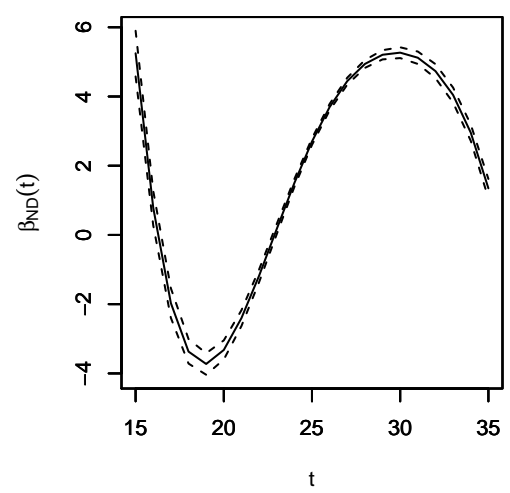

图 4: 武汉市新增确诊人数模型中变系数的估计及置信区间。(a) 变系数 $\beta_{N N}(t)$ 的 $95 \%$ 置信区 间; (b) 变系数 $\beta_{T N}(t)$ 的 $95 \%$ 置信区间; (c) 变系数 $\beta_{N D}(t)$ 的 $95 \%$ 置信区间; (d) 当 $t \in(20,55)$, (a) 的放大图; (e) 当 $t \in(0,35)$, (b) 的放大图; (f) 当 $t \in(15,35)$, (c) 的放大图。

月 30 日降低至 1.32 后增长，在 2020 年 2 月 4 日达到最大值 2.57 , 并于 2020 年 2 月 20 日达 到第二个极值点 0.95 。而湖北省其他 16 个城市的再生数在 2020 年 2 月 5 日增加到 1 , 第二个 极值点 0.68 出现在 2020 年 2 月 17 日。

具体来看，武汉市实时再生数 $R_{1 t}$ 首次开始降低的时间（2020 年 1 月 26 日）与武汉市开 始 “封城” 的时间点基本一致。同时，根据式(3)中 $E\left(d Y_{k t}\right)=E\left(d W_{k, t-m}\right)$ 的假定以及 2020 年 2 月 13 日确诊标准的调整， 2 月 4 日新增的 $d W$ 例感染患者可能于 2 月 13 日被确诊。因此, 迅速增长的新增确诊人数 $(d Y)$ 使得武汉市实时再生数的最大值出现在 2020 年 2 月 4 日。另 一方面, $R_{1 t}$ 的定义表明第 $t$ 天的再生数还依赖于第 $t-m_{1}-1$ 天前感染 COVID-19 病毒的总 人数。由于感染 COVID-19 病毒的总人数在 2020 年 2 月 13 日急剧增长, 因此武汉市的再生数 大约在 8 天后达到第二个极值。但此时疫情逐渐趋于稳定，新增确诊病例也得到了有效的控制。 因此，武汉市的再生数 $R_{1 t}$ 在 2020 年 2 月 20 日达到极值但不超过 1 。同理，湖北省其他 16 个 市实时再生数的变化趋势与武汉市一致且几乎是同步的。 
(a)

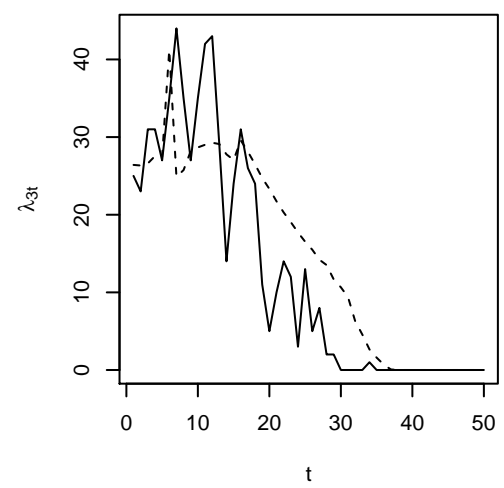

(d)

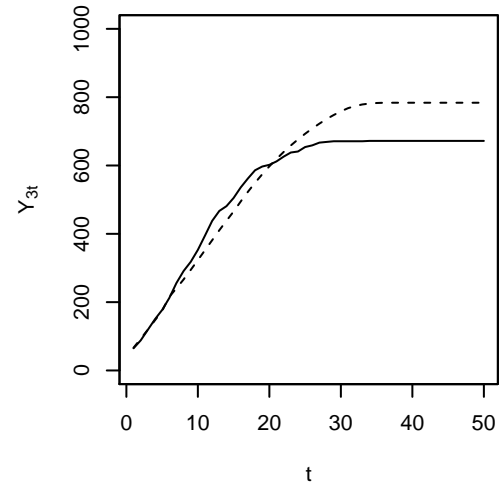

(b)

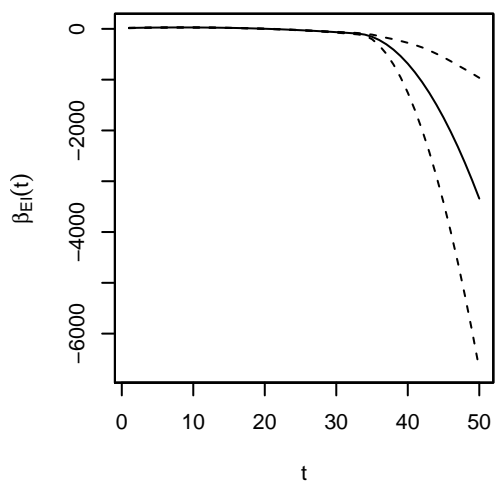

(e)

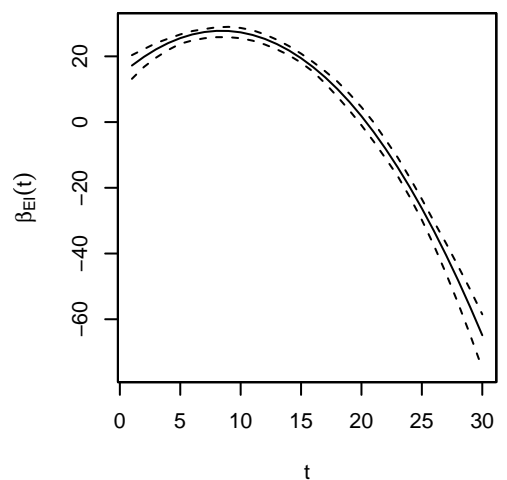

(c)

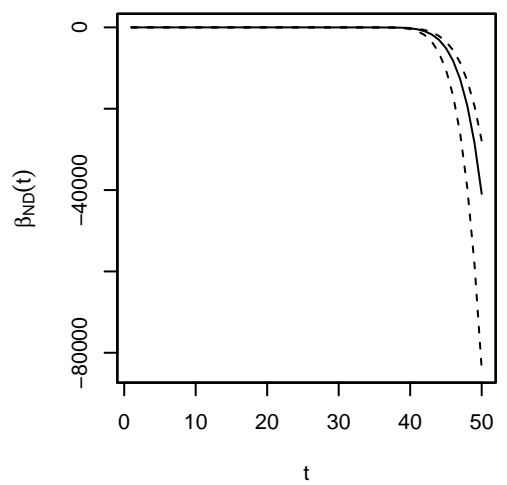

(f)

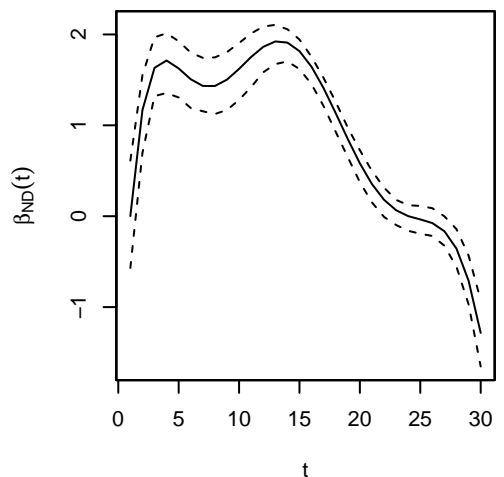

图 5: 除武汉市外湖北省其他城市新增确诊人数模型中变系数的估计及置信区间。(a) 分别用实线 和虚线表示观测到的 $\lambda_{3 t}$ 和估计的 $\lambda_{3 t}$; (b) 变系数 $\beta_{E I}(t)$ 的 $95 \%$ 置信区间; (c) 变系数 $\beta_{N D}(t)$ 的 $95 \%$ 置信区间; (d) 分别用实线和虚线表示观测到的 $Y_{3 t}$ 和估计的 $Y_{3 t}$; (e) 当 $t \in(0,30),(\mathrm{b})$ 的放大图; (f) 当 $t \in(0,30)$, (c) 的放大图。

\section{4 结论与不足}

本文主要评估了交通限制、调整确诊标准、调配医疗资源以及提升核酸检测能力等公共卫 生干预措施对湖北省新冠肺炎疫情防控的影响。论文对武汉市和湖北省其他 16 个城市的新增确 诊人数建立广义半变系数模型，并基于模型结果分别估计了武汉市和湖北省其他城市的潜伏期、 实时传播率以及实时再生数。本文估计出 COVID-19 在武汉市和湖北省其他城市的潜伏期分别 为 8 天和 6 天。模型(1)中系数的估计表明武汉市 “封城” 和确诊标准的调整这两项措施从根 本上抑制了疫情的传播，对控制新冠肺炎疫情在武汉市进一步蔓延起到了至关重要的作用。模 型(1)和(2)的估计结果表明，交通限制、调整确诊标准、调配医疗资源以及提升核酸检测能力的 措施对于抑制 COVID-19 在湖北省的传播发挥了积极作用, 其影响在不同阶段是变化的。且对 于湖北省其他地区而言，医护人员增援和迁徙强度对于疫情防控影响的变化趋势是一致的。同时 本文对实时再生数的相关研究也表明 COVID-19 病毒传播率在武汉市及湖北省其他地区的变化 基本是同步的。 
(a)

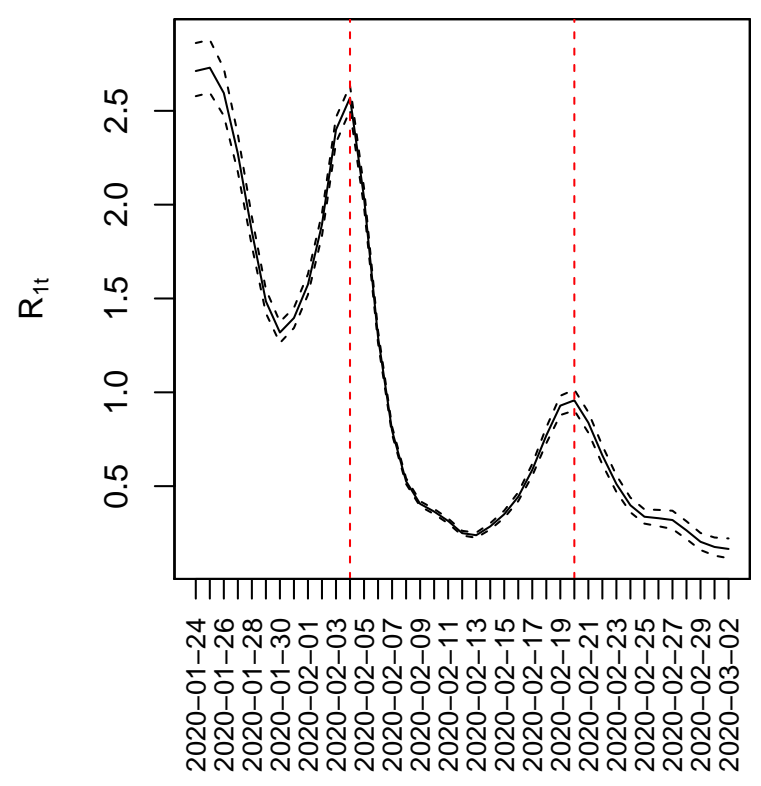

(b)

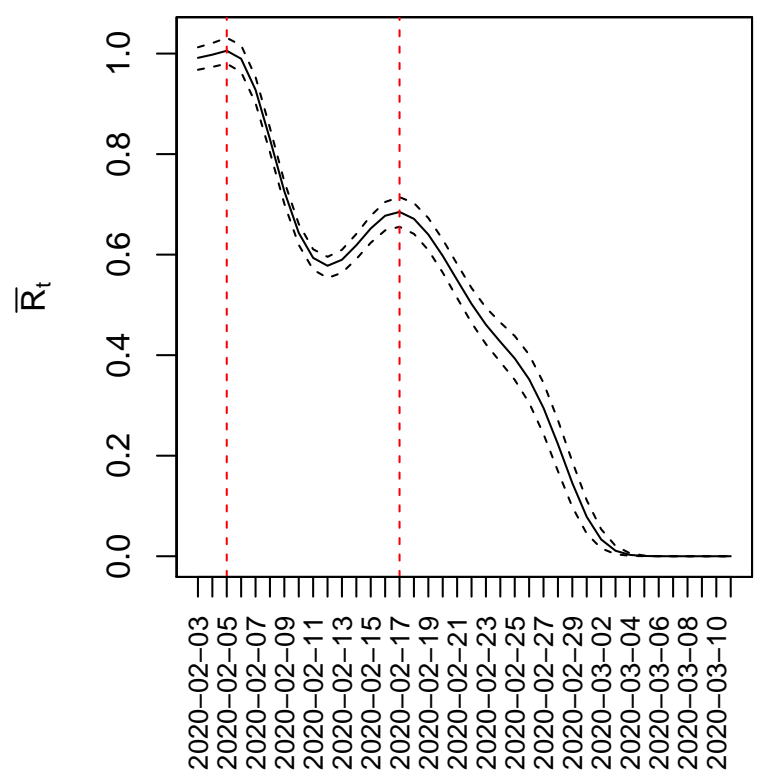

图 6: 实时再生数的估计及 $95 \%$ 置信区间。(a) 武汉市实时再生数的估计及 $95 \%$ 置信区间; (b) 除武汉市外湖北省其他市实时再生数的估计及 $95 \%$ 置信区间。

本研究还存在一些不足。首先, 本文建立的模型尚未考虑城市人口密度、距武汉市的距离以 及当地医疗资源的情况等因素，但事实上这些因素可能对 COVID-19 疫情在湖北其他地区的传 播和控制有一定的影响。其次，对于死亡率和治愈率还需进一步的研究。重症监护病房（ICU） 的数量，体外膜肺氧合（ECMO）的数量以及其他的医疗资源可能会显著影响死亡率和治愈率。 因此，还需收集和整理相应的数据做进一步的分析。

\section{致谢}

本文获得国家自然科学基金项目 “非参数独立性检验及其应用研究” (11971478)，教育部 人文社会科学重点研究基地重大项目《基于大数据的精准医学生物统计分析方法及其应用研究》 (16JJD910002)，中国人民大学公共健康与疾病预防控制交叉学科重大创新平台建设成果资助。

\section{参考文献}

赵培信, 2013. 广义变系数模型的 B 样条估计及 R 软件实现. 经济研究导刊(1): 231-233.

Backer JA, Klinkenberg D, Wallinga J, 2020. Incubation period of 2019 novel coronavirus (2019$\mathrm{nCoV}$ ) infections among travellers from Wuhan, China, 20-28 January 2020. Eurosurveillance, 25(5): 2000062.

Chen Z, Zhang Q, Lu Y, Guo Z, Zhang X, Zhang W, Guo C, Liao C, Li Q, Han X, Lu J, 
2020. Distribution of the COVID-19 epidemic and correlation with population emigration from Wuhan, China. Chinese Medical Journal, 133(9).

Chiang CT, Rice JA, Wu CO, 2001. Smoothing spline estimation for varying coefficient models with repeatedly measured dependent variables. Journal of the American Statistical Association, 96(454): 605-619.

Du Z, Wang L, Simon C, Xu X, Xianwen W, Cowling BJ, Meyers LA, 2020. Risk for Transportation of Coronavirus Disease from Wuhan to Other Cities in China. Emerging Infectious Diseases, 25(5): 1049-1052.

Efron B, 1985. Bootstrap confidence intervals for a class of parametric problems. Biometrica, 72(1): $45-58$.

Efron B, 2003. Second thoughts on the bootstrap. Statistical Science, 18(2): 135-140.

Hastie T, Tibshirani R, 1993. Varying-coefficient models. Journal of the Royal Statistical Society: Series B (Methodological), 55(4): 757-779.

Hoover DR, Rice JA, Wu CO, Yang LP, 1998. Nonparametric smoothing estimates of timevarying coefficient models with longitudinal data. Biometrika, 85(4): 809-822.

Huang JZ, Shen H, 2004. Functional coefficient regression models for non-linear time series: A polynomial spline approach. Scandinavian journal of statistics, 31(4): 515-534.

Kraemer MUG, Yang CH, Gutierrez B, Wu CH, Klein B, Pigott DM, Louis dP, Faria NR, Li R, Hanage WP, Brownstein JS, Layan M, Vespignani A, Tian H, Dye C, Pybus OG, Scarpino SV, 2020. The effect of human mobility and control measures on the COVID-19 epidemic in China. Science, 368(6490): 493-497.

Lin H, Liu W, Gao H, Nie J, Fan Q, 2020. Trends in transmissibility of 2019 novel coronavirusinfected pneumonia in Wuhan and 29 provinces in China. Available at SSRN. http://dx.doi. org/10.2139/ssrn.3544821.

Rice JA, Silverman BW, 1991. Estimating the mean and covariance structure nonparametrically when the data are curves. Journal of the Royal Statistical Society: Series B (Methodological), 53(1): 233-243.

Schumaker L, 2007. Spline functions: Basic theory. Cambridge University Press.

Tian H, Liu Y, Li Y, Wu CH, Chen B, Kraemer MUG, Li B, Cai J, Xu B, Yang Q, Wang B, Yang P, Cui Y, Song Y, Zheng P, Wang Q, Bjornstad ON, Yang R, Grenfell BT, Pybus OG, Dye C, 2020. An investigation of transmission control measures during the first 50 days of the COVID-19 epidemic in China. Science, 368(6491).

Tuite AR, Fisman DN, 2020. Reporting, epidemic growth, and reproduction numbers for the 2019 novel coronavirus (2019-nCoV) epidemic. Annals of Internal Medicine, 172(8): 567-568.

Wu CO, Chiang CT, Hoover DR, 1998. Asymptotic confidence regions for kernel smoothing of a varying-coefficient model with longitudinal data. Journal of the American Statistical Association, 93(444): 1388-1402.

Wu JT, Leung K, Leung GM, 2020. Nowcasting and forecasting the potential domestic and 
international spread of the 2019-nCoV outbreak originating in Wuhan, China: A modelling study. The Lancet, 395(10225): 689-697.

Zhao S, Zhuang Z, Cao P, Ran J, Gao D, Lou Y, Yang L, Cai Y, Wang W, He D, Wang MH, 2020. Quantifying the association between domestic travel and the exportation of novel coronavirus (2019-nCoV) cases from Wuhan, China in 2020: A correlational analysis. Journal of Travel Medicine, 27(2): taaa022. 